\title{
Las wavelets en el análisis de ECG
}

\section{Wavelets in the analysis of $\mathrm{EKG}^{1}$}

\author{
Jose Rodrigo González, Ricardo López y Álvaro Jaramillo \\ Facultad de Ciencias Básicas, Universidad Tecnológica de Pereira, Pereira, Risaralda \\ jorodryy@utp.edu.co
}

\begin{abstract}
Resumen- En este documento se presenta el proceso de reconstrucción de una señal electrocardiográfica aplicando la transformada Wavelet Daubechies a diferente escala, en particular en $j=4$ y $i=6$. Esta señal es descompuesta como suma directa de espacios, a los cuales llamaremos espacios Wavelets $\left(W_{j}\right)$ y espacio de escala $\left(V_{j}\right)$, los primeros dan información acerca de los detalles y el segundo espacio nos permite obtener información del comportamiento de la señal.
\end{abstract}

El objetivo esencial de este análisis es detectar los complejos QRT e intervalos QT, que son partes importantes en el electrocardiograma (ECG), ya que es allí donde se concentra la mayor cantidad de energía de la señal. Los cálculos se realizaron con ayuda del programa MATLAB 8.0, con los que cuenta los laboratorios de ingeniería física de la Universidad Tecnológica de Pereira.

Palabras clave - Espacios L2(R), Función de escala, Wavelet Daubechies, promedio cero, ortogonal, producto interno.

Abstract-This paper presents the process of reconstruction of an ECG signal using Daubechies wavelet transform at different scales, in particular $j=4 \mathbf{j}=6$. This signal is decomposed as a direct sum spaces, spaces which we call Wavelets $\left(W_{j}\right)$ and space scale $\left(V_{j}\right)$, the first giving information about the details and the second space allows us to obtain information on the behavior of the signal.

The main objective of this analysis is to detect the complex QRT and QT intervals, which are important parts in the electrocardiogram (ECG), and that is where the highest concentration of signal energy. Calculations were performed using the program MATLAB 8.0, which has laboratorios in engineering physics from the University of Pereira.

1 J.R. González, Ph.d, docencia en el Departamento de Matemáticas, de la Universidad Tecnológica de Pereira, Pereira (Colombia); email: jorodryy@utp.edu.co.

R. López, Ms.C, docencia en el Departamento de Física, Universidad Tecnológica de Pereira, Pereira (Colombia); email: rilopez@utp.edu.co.

A. Jaramillo, Ms.C, docencia en el Departamento de Matemáticas, Universidad Tecnológica de Pereira, Pereira (Colombia); email: aljamil@utp.edu.co.

Fecha de Recepción: 30 de septiembre de 2015

Fecha de Aceptación: 18 de septiembre de 2016
Key Word - Space L2(R), Scale function de, Haar, Wavelet, zero average, orthogonal, inner product..

\section{INTRODUCCIÓN}

Una de las motivaciones para realizar este trabajo fueron las cifras publicadas por el Ministerio de la Protección Social en lo referente a la salud cardiaca de los Colombianos, donde se muestra que durante los años 1990 a 2001 las enfermedades cardiovasculares ocasionaron el $24 \%{ }^{2}$ del total de las muertes registradas en el país, ocupando el primer lugar en mortalidad, por encima de los accidentes de tránsito y los homicidios, situación que se ha convertido en un problema de salud pública. Es por esta razón que el objeto de estudio presentado en este trabajo es el análisis de electrocardiogramas y las dificultades presentadas por los especialistas para realizar diagnósticos y tratamientos en pacientes que residen en municipios apartados de la geografía nacional.

Este documento propone un método alternativo para el análisis de electrocardiogramas ${ }^{3}$ usando la transformada wavelet e implementando una aplicación en el software matlab 8.0, que permite que un cardiólogo pueda analizar la información de pacientes que se encuentren a muchos kilómetros de distancia y pueda realizar un adecuado diagnóstico y tratamiento, enviando esta información por la internet.

Para iniciar con el proceso, primero se indagó sobre la forma en que se realiza el estudio o análisis de los ECG en la actualidad, con gran sorpresa se encontró que existen diversos métodos alternativos que permiten realizar el análisis de éstos. Durante el proceso de recolección de información sobre la teoría Wavelet y sus aplicaciones resultó inevitable encontrar

\footnotetext{
${ }^{2}$ Cifras tomadas de la página del ministerio de la protección social www. minpro-tecciónsocial.gov

3 Para realizar este estudio se utilizó la base de datos certificada de la página de phys-ionet.org. El estudio se realizó en los laboratorios de la facultad de ingeniería física de la Universidad Tecnológica de Pereira, que cuenta con la licencia del software MATLAB 8.0
} 
numerosa y valiosa información sobre esta, así como múltiples trabajos en diversas ramas de la ciencia. En nuestro caso, la aplicación de las Wavelets en el proceso de detección de los complejos QRS, ondas T y S e intervalos QT, como ya se mencionó, se encontraron tres métodos alternativos para realizar análisis de electrocardiogramas: El método cadenas de Markov, El método del módulo Máximo, y el algoritmo de Pan-Tompkins.

El método propuesto en este documento centra todo su estudio en la detección de los complejos QRS y los intervalos QT, que son los puntos más importantes de ECG, pues es alli ${ }^{4}$ donde se concentra la mayor información del registro eléctrico de la actividad cardiaca de los pacientes; éste resultado se obtiene cuando se realiza el análisis wavelet en diferente escala de resolución [1].

\section{CONTENIDO}

\section{A. Aplicación y análisis de electro ECG}

El análisis de electrocardiogramas que se presenta a continuación aplica la transformada Wavelet, usando la Wavelet Daubechies [7] a diferente escala, 4 y 6. Por tal motivo es necesario definir los coeficientes Wavelet y de escala.

Para realizar este análisis y su aplicación en el programa Matlab 8.0, primero se define la transformada Wavelet discreta como:

$$
W_{j, k}=\int_{-\infty}^{+\infty} x(t) \psi_{j, k}(t) d t
$$

Donde $\boldsymbol{\psi}_{\boldsymbol{j}, \boldsymbol{k}}(\boldsymbol{t})$ es una base Wavelet ortonormal, que nos permitiría reconstruir la señal original.

Los coeficientes de escala se definen como:

$$
S_{j, k}=\int_{-\infty}^{+\infty} x(t) \phi_{j, k}(t) d t
$$

Siendo $j$ la escala y $k$ la localización en tiempo. En esta aplicación la señal discreta de entrada $S_{0, n}$ es finita, de longitud $N$, el cual es una potencia entera de $2: N=2^{J}$, de tal manera que el rango de la escala esta determinado por el intervalo $0<j<J$.

La aproximación discreta de la señal tiene la forma:

$$
x_{0}(t)=x_{J}(t)+\sum_{j=1}^{J} d_{j}(t)
$$

\footnotetext{
${ }^{4}$ La notación que se presenta en este trabajo, es tomada del autor Mallat Stephane. A wavelet tour of signal processing. Segunda edición 2
}

Donde la medida de la señal aproximada en una escala $J$ es:

$$
x_{J}(t)=S_{J, k} \phi_{J, k}
$$

y los detalles de la señal de aproximación correspondientes a una escala $j$ se define por:

$$
d_{j}(t)=\sum_{k=0}^{2^{J-j}-1} W_{j, k} \psi_{j, k}(t)
$$

La aproximación de la señal deseada resulta de la combinación de los coeficientes de aproximación con los coeficientes de detalle, es decir:

$$
x_{j}(t)=x_{j-1}-d_{j}(t)
$$

El análisis propuesto desde Matlab se puede resumir siguiendo los siguientes pasos:

i. Se visita la página http://www.physionet5.org/

ii. Se selecciona el archivo (Registro100) a estudiar.

iii. Se transforma estos archivos a .mat, usando [A2.5]

iv. Desde matlab se corre el programa [A2.5]

\section{Análisis de dos electrocardiogramas, usando la transformada wavelet.}

Las figuras 1 y 2 muestran el análisis 6 de un electrocardiograma o registro, el número 100, tomados de la base de datos de physionet. La primera columna presenta el análisis a diferente escala de los coeficientes de aproximación o de escala y la segunda registra el análisis a diferente escala de los coeficientes Wavelets o de detalle. En este estudio se observa que con los coeficientes de detalle se obtiene una información muy precisa de los QRS, particularmente en la escala $\mathrm{j}=4$, seguido hacia abajo, primero se muestra el ruido de la señal (aproximación 1), luego se evidencia que al aumentar la escala se obtiene una mejor resolución (relación inversa entre la escala y la frecuencia) del ECG, es decir las frecuencias altas desaparecen, además aparece el efecto zoom.

\section{Análisis de dos electrocardiogramas, usando la transformada wavelet con ruido de $60 \mathrm{~Hz}$.}

La propuesta en esta parte del trabajo radica en incorporar un ruido de $60 \mathrm{~Hz}$ a los electrocardiogramas, 100 y 101 figura 3 y 4 detectar los QRS, cambiando el nivel de resolución de 4 a 6. La selección de esta frecuencia tiene una gran importancia, ya que la mayoría de los equipos electrónicos de un laboratorio afectan a otros con una frecuencia de 60 Hz. 


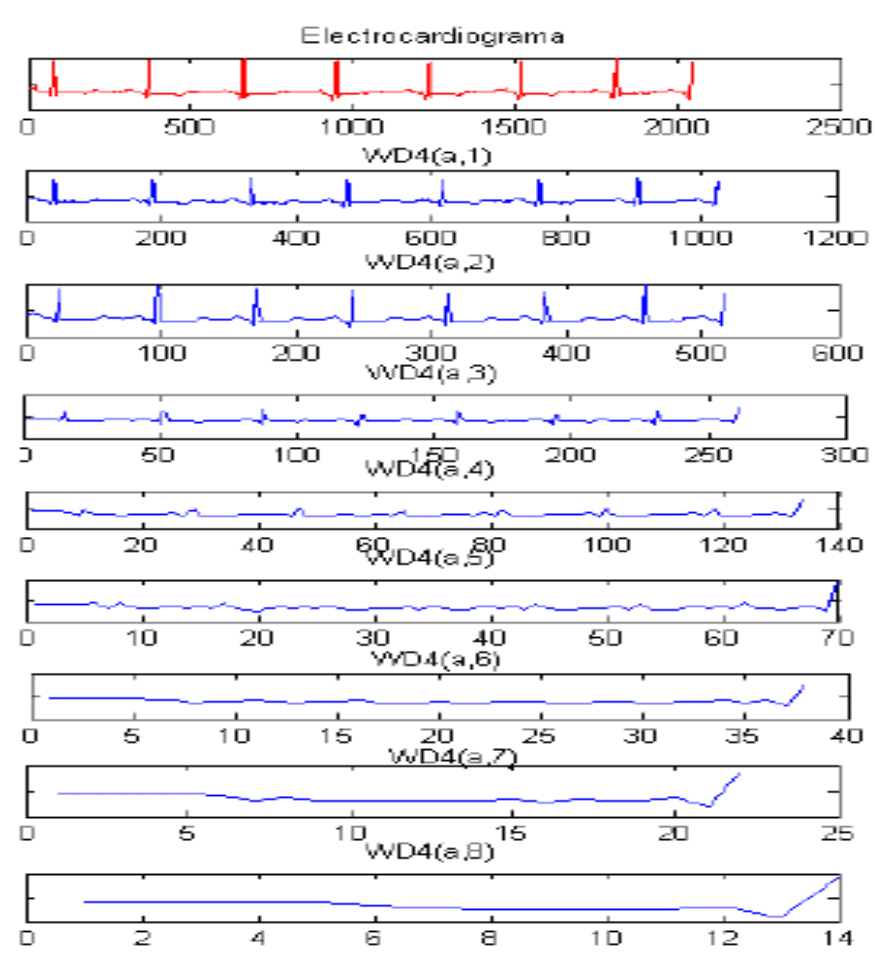

Figura 1. Análisis Multiresolución del EGC

(Registro 100), usando Wavelet Daubechies 4.

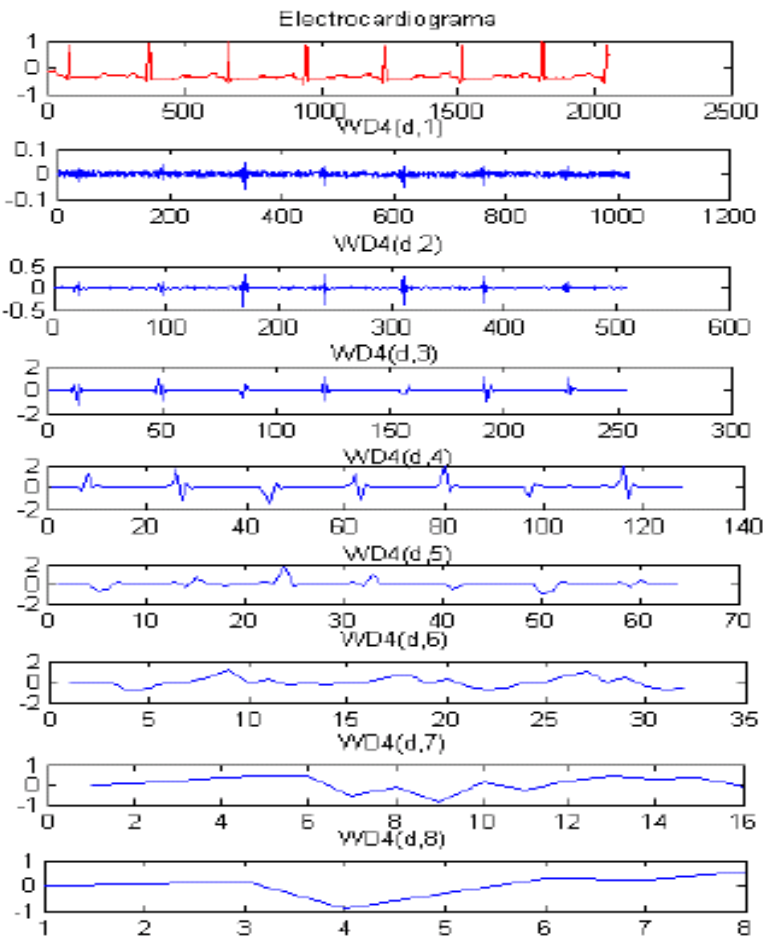

Figura 2. Análisis Multiresolución del EGC (Registro 100), usando Wavelet Daubechies 4.

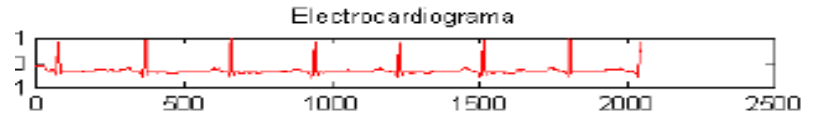

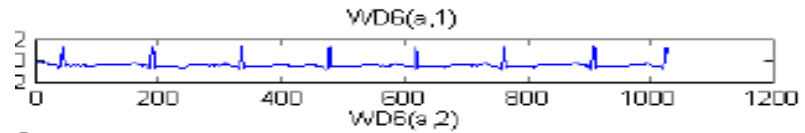

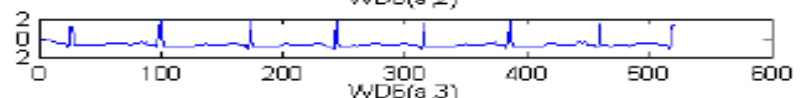

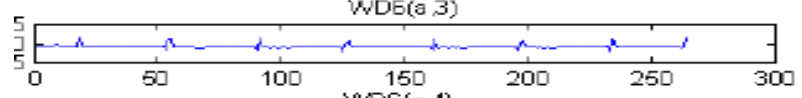

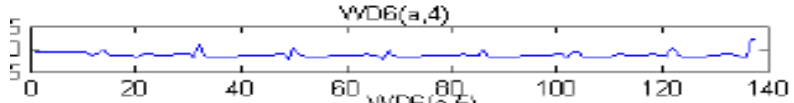
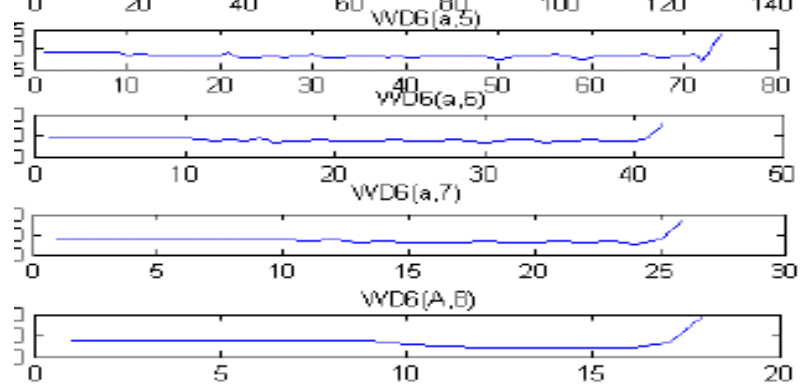

Figura 3. Análisis Multiresolución del EGC (Registro 100), con ruido de 60 $\mathrm{Hz}$, usando Wavelet Daubechies 6.

\section{RESULTADOS}

Después de realizar el análisis Wavelet en la detección de los QRS de electrocardiograma, mostrado en el capítulo anterior, se presenta el cálculo y análisis del error que surge de dicho estudio cuando se aplica esta transformada a esta señal. El error que se presenta en este trabajo es el error cuadrático medio definido como:

$$
\boldsymbol{E}_{c m}(\boldsymbol{f})=\sqrt{\frac{\sum_{i=1}^{n}\left(e_{i}\right)^{2}}{n}}
$$

Siendo:

$$
e_{i}=y_{i}-f\left(x_{i}\right)
$$

Donde:

\section{$y_{i}:$ Es la señal de entrada (ECG original) \\ $f\left(x_{i}\right)$ : Señal de respuesta (ECG después de aplicar la transformada Wavelet)}

Para calcular este error se tomó una muestra de 29 archivos de una población de 120 archivos disponibles de la página de physionet. 


\section{A. Estadísticos del error cuadrático medio}

A continuación se calcula el error cuadrático medio y la desviación estándar para los 29 ECG, este proceso arroja los siguientes resultados exhibidos en la tabla 1.

La información presentada en esta tabla consiste en un arreglo rectangular de 29 columnas (ECG) y dos filas, que corresponden al error cuadrático medio y la desviación estándar respectivamente. La segunda columna corresponde al electrocardiograma 100, cuyo error cuadrático medio es 0.0259 (Voltios), que se obtiene de las diferencias entre los vectores: ECG (entrada) y ECG que resulta del análisis wavelet a escala $j=4$ y luego se determina la desviación con respecto al $E_{c m}$. El proceso se repite para los demás archivos restantes.

El análisis estadístico que se realizó, demuestra la efectividad de la aplicación del análisis Wavelet en la lectura de electrocardiogramas, particularmente en la detección de los complejos QRS. Con los datos obtenidos y registrados en la tabla 9 se realizaron las siguientes pruebas: histograma y prueba gráfica QQ-plot normal. En la aplicación de estas pruebas se utilizó el programa estadístico ${ }^{5}$ SPSS e INFOSTAT.

\begin{tabular}{|l|l|}
\hline Tamaño de la muestra ( $\mathrm{n}$ ) & 29 \\
\hline Media (ECM -promedio) & 0.022 (Voltios) \\
\hline Desviación Estándar & 0.010 (Voltios) \\
\hline Coeficiente de Variación & $43 \%$ \\
\hline Error Mínimo & 0.007 (Voltios) \\
\hline Error Máximo & 0.043 (Voltios) \\
\hline
\end{tabular}

Tabla 1. Resumen del análisis estadístico de la implementación de la transformada Wavelet Daubechies $j=4$.

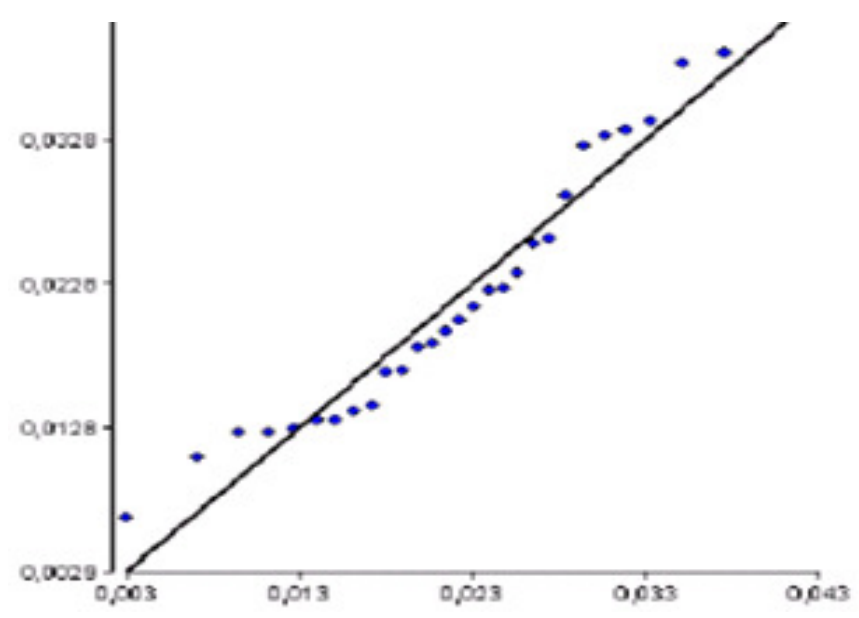

Figura 5. Gráfico Q-Q PLOT correspondiente a los errores cuadráticos medios utilizando como distribución teórica la distribución normal.

${ }^{5}$ Los programas SPPS e INFOSTAT son programas estadísticos disponibles (Gratuitos) en internet.

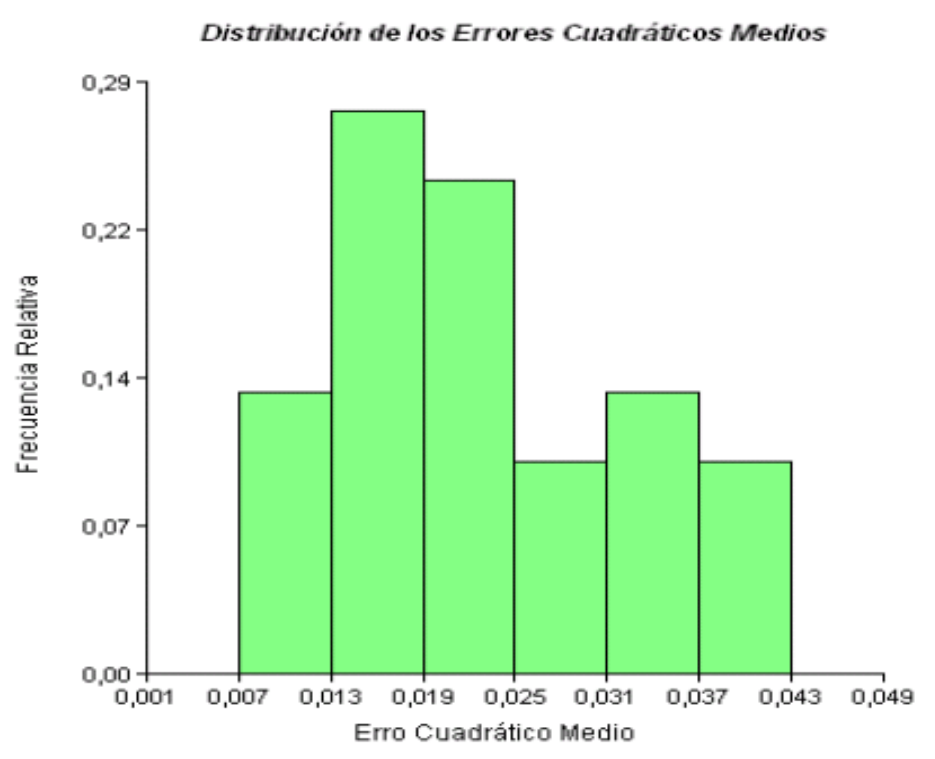

Figura 6. Histograma muestra la distribución de los 29 errores cuadráticos medios.

\section{CONCLUSIONES}

Del trabajo que se realizó se obtuvieron las siguientes conclusiones:

- El análisis Wavelets reúne toda la información de una señal en un solo plano tiempo-frecuencia, hecho que resulta de gran importancia cuando se analizan señales de frecuencia variable o que cambian bruscamente en el tiempo.

- Se detectaron los complejos QRS, usando la Wavelets Daubechies haciendo el análisis a diferente escala de resolución, particularmente en $j=4$ y usando la wavelet Daubechies 2.

- El análisis de electrocardiogramas usando la transformada Wavelet permitió identificar enfermedades del corazón como las arritmias y la fibrilación Auricular.

- La Wavelet Daubechies cuando se aplicó a un electrocardiograma en diferente escala produjo un efecto filtro, eliminó ruido, es decir se evidencia la relación inversa entre la frecuencia y la escala, a escalas bajas frecuencias altas y a escalas altas frecuencias bajas.

- El método expuesto en este trabajo detectó los puntos QRS de un electrocardiograma con un error cuadrático medio de 0.022 (Voltios), con un coeficiente de variación del $43 \%$, lo cual indica una gran variabilidad de los errores, es decir estos no son 
homogéneos, a pesar de ser valores pequeños (cercanos a cero).

- La gráfica de los errores cuadráticos medios obtenidos con el análisis wavelet, muestran un comportamiento similar a una distribución normal, cuya media es de 0.022(Voltios) y varianza de 0.00009 (Voltios), resultados que permitirán utilizar el modelo teórico de la distribución de probabilidad normal, para determinar errores en modelaciones futuras, con base en el estudio presentado en este trabajo.

- El análisis de Fourier no pierde vigencia con la aparición de la teoría Wavelets, por el contrario, es un complemento, puesto que esta permite analizar una gama de funciones más amplia, en la cual encontramos funciones que varan abruptamente con el tiempo.

\section{RECOMENDACIONES}

En el desarrollo del trabajo y la presentación matemática aparecen algunas series de infinitos términos, cálculos que resultan difíciles de procesar, es por esta razón que es indispensable implementar algunos programas (programación orientada a objetos) en Matlab, que permitan mostrar mediante aplicaciones sencillas los aspectos más relevantes de la teoría Wavelets.

\section{REFERENCIAS}

[1]. Mallat, S. A wavelet tour of signal processing, Ecole Polytechnique, Paris Courant Institute, New York Uni 1998

[2].Plaumann, E y Unger, H.Analisis funcional I, Editorial Alhambra, S.A,Madrid, 1974.

[3]. Kreyszig, E.Introductory functional analysis withaplications, Universidad de Windsor. Editorial John Wiley and Son. Canada (1978)

[4]. Daubechies Y. Ten lectures on Wavelets, ed. Society for industrial and applied mathematics, 1992

[5]. Irarrazaval, P.Analisis de señales, Pontificia Universidad Católica de Chile.Editorial Mcgraw hill, (1999)

[6]. Rivlin, Theodore. An introduction to the aproximations of functions. Dover publications, inc. New York, (1964)

[7]. J.S. Sahambi, S.N. Tandon,R.K.P. Bhatt. Using Wavelet Transforms for ECG Characterization. IEEE engineering in medicine and biology, jan-uary/fabruary 1997.

[8].A. Mendoza, L. Archila, J.A. Ardila.Caracterización del Intervalo QT en una señal electrocardiográfica usando la Transformada Wavelet. II Memorias congreso Latinoamericano de ingeniería biomédica, Habana 2001, Mayo 23 al 25, 2001, La habana, Cuba.

[9]. Mallat S: Zero crossings of wavelet transform, IEEE Trans. Information. Theory, 37, No, 4, 1019-1033, july 1991.

[10]. MIT/BIH arrhythmia database -Tape directory and format spectification. Document BMEC TR00, mass. Inst. Technol, Cambridge, 1980. Database is availa from Bioengineering
Division KB-26, beth Israel Hospital, Brooline Avenue, Boston, MA 02215. 330

[11]. R López y A Jaramillo, Procesamiento de señales usando Wavelet. Tesis de Grado, Maestría en enseñanza de las Matemáticas, Universidad Tecnológica de Pereira. Febrero 2 de 2011. 